\title{
Research methodology in the postinformatic age
}

\author{
ALAN LESGOLD \\ University of Pittsburgh, Pittsburgh, Pennsylvania
}

\begin{abstract}
The widespread availability of computers and powerful software as commodities should offer new approaches to research methodology that both take advantage of extant tools and address new research questions prompted by the broad penetration of informatic resources into our society.
\end{abstract}

It is an honor to have shared the leadership of the Society for Computers in Psychology with such pioneers as Tepas, Sidowski, and Castellan. Accordingly, I am pleased to contribute to this commemorative discussion.

When I attended my first meeting of what was then the "on-line" conference, computer resources for psychological research were still extremely limited and quite exotic. A web of personal and professional relationships had been required to get me time-shared access, by network, to a machine at the other end of the country that had less power than the one on my desk today (and I have a pretty ordinary PC on my desk). My memories are of clever hand-built peripherals, unending numbers of papers on each new researcher's personal effort to write a subroutine to compute chi-square values, visionary efforts to develop computers for education, continuing discoveries that experiments once thought impossible to manage could now be performed, seeing that most everyday system tools on the system that I was using were written by a Nobel laureate biochemist who needed them to get his work done, and suspecting that many of the less imaginative colleagues who were previously seen fiddling with equipment racks in the animal labs were now fiddling with personal computers. It was an exciting but chaotic time, with portents of a great new era for psychology but also of the computer as a multiplier of mediocrity as well as genius. On balance, we have done well.

Today, most of the tools used by the average psychologist are commodities, in the sense that they are cheap and that the general ability to use them is becoming as embedded in our culture's shared knowledge as is the ability to make a long distance call. Furthermore, most laboratory paradigms can best be handled by powerful psychological laboratory software, some of which has been pioneered by this Society's members. We do not need an annual conference to tell us how to do things that are well documented in the manuals and texts that are starting to accompany such software. We could go out of business as a Society, but I think that there are important new needs that we ought to address.

Correspondence should be addressed to Alan Lesgold, Learning Research and Development Center, University of Pittsburgh, Pittsburgh, PA 15260.
As pioneers of the use of information technology in psychology, we know some things that are very importantabout the changes that technology has made possible in our field, about the preparation that a psychologist needs to take full advantage of the new tools, and about the broader impact of those tools on our society. These things that we know lead to several conclusions about where we should next proceed as members of psychology's technology subculture.

One responsibility we have is to be honest with those who would enter our profession. Powerful information processing tools afford new opportunities for tightly coupling the complexities of cognitive activity to strong formal theories. We cannot continue to allow our students to believe that they can make significant contributions to psychological science without an adequate education in mathematics, computing and information science, and the physical and biological sciences. Too often, our students are bright, highly verbal people who have passed the college math requirement by taking high school calculus, satisfied their science requirement with relatively general psychology courses, and perhaps taken one course in programming in high school or college. Beyond that, they often have no background in the formalisms that every science uses to express its theories, and they lack the facility with computational tools that would allow them to fully exploit the computer as a partner in research. Surely, it is time to look to our sister sciences for examples of how to prepare those who will eventually join us as colleagues. A biology major often requires more math, chemistry, and other science courses than biology courses. As we merge cognitive and brain sciences more and more, we must attend to the striking disparity between the preparation for entry into the sciences of the mind and that for entry into the sciences of the body. If we do not, psychology will wither as a science.

Going beyond the new cognitive competences needed by the psychologist in the postinformatic era, we should also reflect on what will be needed more generally by one who would be a full and valued participant in a society that takes computers for granted and leverages them broadly. It is a cliché that the computer has changed life as much as the steam engine, but we seldom reflect on what this means. In the past, once machines had come to provide the routine means of doing hard work, people 
came to be evaluated less for their physical prowess and more for their ability to harness machines to get things done. Physical competence did not disappear as a valued capability; we still have carpenters, plumbers, roofers, and many others on whose physical capability we depend. However, planning, problem-solving, record-keeping, numerical computation, and a host of other cognitive skills have become more important because they are necessary in the harnessing of mechanical power to accomplish social needs. Much of cognitive psychology consists of the study of just those competences that became more valuable with the industrial age.

Now that we have entered the information age, we have seen a rapid decline of jobs that embody the execution of relatively fixed algorithms, whether symbolic (as in a job like bookkeeping) or physical (as in being a worker on an auto assembly line). Computers and robots do the repetitive work once done by people. The jobs that remain for those who lack a broader set of cognitive competences are often marginal, with little opportunity for personal development, poor wages, and significant competition from a world surplus of unskilled labor. Complex cognitive skills are increasingly valued, and there is a range of new social and cognitive capabilities that are particularly important.

Because our lives are so multifaceted and complex, especially in the business world, intelligence must often be distributed. Human business activity routinely involves groups of people who split up complex cognitive tasks so that each person can specialize in a manageable knowledge domain. However, for a variety of business purposes, people with different views of a task situation must interact. To interact successfully, they must be able to negotiate a shared situation model quickly and agree on how they will talk about the situation that they face. This may happen in a simple face-to-face discussion, but it may also occur over a variety of communications media with qualitatively and quantitatively differing bandwidths. The kinds of activities that I have in mind include both high-level meetings of major corporate decision-making committees and mundane activities such as customer-service representatives' interacting with stymied customers. They also include group decisions about military threats, factory production breakdowns, power-plant emergencies, environmental disasters, and other high-stakes interactions. The high-technology world, driven by computer power, has created a great demand for the skills that underlie successful performance in such situations, and we as psychologists need to begin studying them.

To do this, we will need new laboratory technology. For example, the situation of different people confronting a complex situation from different perspectives and having to resolve partially conflicting viewpoints seems best captured by a network of workstations that can be both the media of communications between research participants and the sources of different inputs to each. This creates a new methodology need.
Strangely enough, even though most psychological experimentation involves the wholesale activity of groups of participants, each often at a separate terminal, there is extremely little use of network technology for multiperson research. Although network applications development tools are still rather limited, it is possible to develop group interaction laboratories, as a small collection of ONR-funded researchers are now doing. From personal experience (John Levine, Kathleen Carley, and I have a small project that is part of the ONR effort just mentioned), I can tell you that such development is possible and that it will become much more common as network development tools improve, something that is taking place rapidly.

Not all research on group cognitive activity will be done with computer terminals. Indeed, some of the best current work involves ethnographic observations of group activity, often in work situations. The data from such studies is generally a combination of field notes and video or audio tapes. For studies of any kind of complex activity, the task of coordinating and analyzing the data is massive. Consequently, there is need for tools to manage the data collection and analysis process. Such tools are starting to appear, although they will need another generation to mature. For example, Jeremy Roschelle and others at the Institute for Research on Learning have developed a system that allows field notes, annotations of video segments, pointers to video segments, transcriptions, and other information to be mapped visually onto a timeline. What has been built is something like a hypermedia spreadsheet in which the rows correspond to consecutive time slices and the columns represent different forms of data and interpretation. Combined with appropriate search tools, the system provides strong support for group discussion and analysis of field protocols. For example, once some preliminary categorizing has been done, the Roschelle tool can be used to search for every video segment that has been marked in a certain way, thus allowing new hypotheses to be quickly tested against rich data. Such search tools will be critically important in scaling up ethnography to the level of replicable analytic detail that psychological researchers demand.

Another area in which methodology and research support each other is interface design. Surely, one secret to the success of some of our own tools, such as the MEL system built by the former Society president Walter Schneider, consists of their powerful and easy-to-use interfaces. When we build tools, we should always be trying to use the best interface technology available. On the other hand, we should also pay attention to what works and try to figure out why it does so. Here again, we who use these tools and follow their development are particularly well placed for studying them, the processes on which they depend, and the ways in which they succeed or fail as a function of design principles that do, or should, rest on sound basic theory.

I end with some more mundane matters. First, I note that a certain level of computer power is becoming quite 
affordable, but that specialized workstations of much higher power remain somewhat expensive. For this and other reasons having to do with networking economics, we will see a wider variety of servers on networks, including both hardware-oriented servers such as FAX servers and software-oriented resources such as databases and specialty processors like natural language generators. Already, we see cases of psychological research efforts that require networking of specialized intelligent computer systems. For example, Blake Ward at Carnegie Mellon University is completing a dissertation project in which the tutorial interface for an intelligent tutoring system was programmed in one language on one kind of computer while the core modeling and instructional decision-making were programmed in a different language on a different computer.

The networked server approach should permit much more frequent use of standardized tools, such as knowledge bases and natural language generators. These tools will permit richer experimentation than was previously feasible. To the extent that they are standardized, they will also permit replicable experimentation, which is critically important to the future of psychological science. Perhaps the best natural language generators, graphic-user interface packages, knowledge-structuring tools, and other such resources are the memory drums and Skinner boxes of our time. Surely psychology needs to return its attention to experimental replicability. 